\title{
Editorials
}

\section{Outcomes in Outpatients - what occurs outside?}

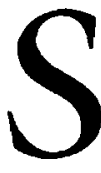

INCE the report in 1909 by James Nicoll ${ }^{1}$ on a series of 8,988 children who were operated upon as day cases, the practice of ambulatory surgery and anaesthesia has grown at an enviable pace. In many Canadian hospitals the $50 \%$ target has been comfortably exceeded and up to $70-80 \%$ of cases are managed on an ambulatory basis. Another trend is also emerging - that of office based anaesthesia. The SMG Marketing Group of Chicago, Illinois estimates that $8.5 \%$ of all operations were office based in 1994 and that this segment is projected to increase to $20 \%$ by the year 2001 . The driving force underlying this trend in favour of ambulatory anaesthesia is the economic payoff. ${ }^{2}$ Cost savings that can be realised for some procedures such as inguinal hernia can be as high as $60 \%$. In addition, attitudes towards hospitalisation have changed: doctors perform surgery less invasively and there has been pressure on provinces to balance budgets. According to Statistics Canada, between 1986-87 and 1994-95 there was a 30\% decline in hospital beds across the country, a $20 \%$ decrease in duration of hospital stay and a $15 \%$ increase in outpatient visits to 38 million. $^{3}$ In spite of this widespread shift towards ambulatory surgery there has been little critical evaluation of the outcome of this service. It is therefore pertinent to enquire whether such a shift in practice is doing any harm.

Preliminary studies for selected diagnostic categories such as transurethral prostatectomy and uterine or adnexal procedures indicate that length of hospital stay decreased by up to $18 \%$ between $1989-90$ to $1992-93$ without affecting quality of care, rates of readmission or number of physician visits. ${ }^{4}$ In many hospitals such procedures are often performed on an outpatient basis but such measures of quality are at present unavailable. Large outcome studies indicate that major complications after ambulatory surgery are uncommon. Reported mortality rates range from 1: 11,273 to 1 : 100,000 with the main causes being myocardial infarction, pulmonary embolism, respiratory failure and cere- brovascular accident. From an epidemiological perspective, the paucity of serious complications makes statistically useful studies difficult.

Minor morbidity has, therefore, become a surrogate measure when assessing outcomes after ambulatory surgery. Methods used to assess minor morbidity include post operative symptoms before and after discharge. The most common symptoms in the first $24 \mathrm{hr}$ after discharge are sore throat (28\%), pain (24\%), headache (12\%), drowsiness (11\%), nausea and vomiting $(7 \%)$ and fever $(5 \%) .{ }^{5}$ These incidences have not changed in the last $20 \mathrm{yr}$ in spite of changes in surgical and anaesthetic practice $!^{6}$ The precise incidences of minor symptoms will vary according to the case mix at each institution. Certain procedures, such as gynaecological laparoscopy and tonsillectomy, are associated with more pain and emesis. High incidences of emesis, sedation and dizziness are associated with the administration of postoperative opioids. Under-treatment of pain and nausea is still evident in many hospitals even though a large proportion of patients experience both. ${ }^{7}$

Unanticipated hospital admission rates are another measure of outcome after ambulatory surgery and help to monitor quality control and provide the feedback essential for determining the appropriate blend of procedures and patient types. Published series report rates from $0.1 \%$ to $9.5 \%$ and hospital based ambulatory surgery units tend to have higher admission rates. The usual reasons for unscheduled admissions are pain, bleeding, emesis, syncope, social and complications of surgery. However, there has been no large scale prospective study that has attempted to identify factors that may be predictive for unanticipated admissions. In the present issue, Fortier and Chung ${ }^{8}$ describe their prospective study of 15,179 outpatients at the Toronto Hospital. The overall rate of unanticipated admission was $1.42 \%$. Ear nose and throat surgery $(18 \%)$ and urology (4.8\%) had the highest rates. Longer procedures (> $\mathrm{l} \mathrm{hr)} \mathrm{and} \mathrm{those} \mathrm{finishing} \mathrm{after} 3 \mathrm{pm}$ were more likely to result in unplanned admission. Postoperative factors 
predictive of unplanned admission were bleeding, pain, drowsiness and, nausea and vomiting. The present study did not find general anaesthesia an important risk factor. Gold $e t a l .{ }^{9}$ found general anaesthesia to be a risk factor with an odds ratio of 5.18. Perhaps a change in anaesthetic practice with the use of short acting agents, such as propofol, account for this difference. Some unexpected relationships have also emerged such as male sex and body mass index. Intuitively, these do not make sense and need further investigation. However, it is apparent that many of the reasons for readmission are social and medical. Both suggest a need for better patient and surgeon education as well as medical optimisation and screening for social support at home.

In conclusion, it is disturbing that so many outpatients report minor symptoms such as sore throat, nausea, headache, drowsiness, pain and backache and report a high rate of dissatisfaction $(65 \%) .{ }^{10} \mathrm{An}$ increasing number of symptoms $24 \mathrm{hr}$ after operation is a predictor of dissatisfaction with anaesthesia and global dissatisfaction with ambulatory surgery. ${ }^{11}$ Failure to treat common symptoms such as pain can lead to severe behavioral changes in children that last up to four weeks after day case surgery. ${ }^{12}$ More recent reports of high infection rates in the outpatient setting are equally worrisome and need to be heeded. ${ }^{13} \mathrm{~A}$ more intensive study of post-discharge sequelae in outpatients is clearly warranted and this should include indices such as return to function and delayed complications after ambulatory surgery in hospital affiliated units as well as free standing facilities. In Canada, no organisation has been charged with implementing a comprehensive proactive approach to quality assurance in the outpatient setting. The potential exists for increased expansion of free standing facilities providing insured services and market-driven expansion of uninsured services. Provincial authorities need to develop a comprehensive framework for addressing the quality of care in all outpatient facilities. ${ }^{14}$

\section{Évolution des patients ambulatoires - Que se passe-t-il à l'extérieur?}

Depuis le rapport de James Nicoll ${ }^{1}$, en 1909, sur une série de 8988 enfants qui ont été opérés comme des cas de jour, la pratique de la chirurgie et de l'anesthésie ambulatoires a progressé à un rythme enviable. Dans de nombreux hôpitaux canadiens, l'objectif de $50 \%$ a été facilement dépassé et il y a jusqu'à 70 à $80 \%$ de cas qui sont traités selon une base ambulatoire. Une autre tendance apparaît aussi - celle de la clinique privée d'anesthésie. Le SMG Marketing Group de Chicago, en Illinois, estime que $8,5 \%$ de toutes les interventions chirurgicales ont été faites dans des cliniques privées en 1994 et on peut s'attendre que cette proportion s'accroisse à $20 \%$ en l'an 2001. Ce qui alimente le courant en faveur de l'anesthésie ambulatoire, ce sont les bénéfices économiques. ${ }^{2}$ Les épargnes réalisées pour certaines opérations comme celle de l'hernie inguinale peuvent s'élever jusqu'à $60 \%$. En outre, les attitudes ont changé face à l'hospitalisation : les médecins pratiquent des interventions moins effractives et on presse les provinces d'équilibrer leur budget. D'après Statistiques Canada, entre 1986-1987 et 1994-1995, il y a eu une baisse de $30 \%$ des lits d'hôpitaux à travers le pays, une diminution de $20 \%$ de la durée de séjour à l'hôpital et une augmentation de $15 \%$ des visites à titre de patients ambulatoires, pour atteindre 38 millions. ${ }^{3}$ En dépit de ce virage considérable vers la chirurgie ambulatoire, il n'y a eu que peu d'évaluation critique de l'évolution de ce service. Il est donc pertinent de chercher à savoir si un tel changement de pratique a eu des effets négatifs.

Les premières études portant sur des catégories diagnostiques. sélectionnées, comme la prostatectomie transurétrale et les interventions utérines ou annexielles, indiquent que la longueur du séjour à l'hôpital a connu une diminution pouvant aller jusqu'à $18 \%$ entre 1989 . 1990 et 1992-1993 sans modifier la qualité des soins, les taux de réadmissions ou le nombre de visites médicales. ${ }^{4}$ Dans de nombreux hôpitaux, des interventions semblables ont été réalisées auprès de patients externes mais les mesures de qualité, mentionnées plus haut, ne sont pas disponibles actuellement. De grandes études indiquent que les complications majeures résultant d'une chirurgie ambulatoire sont peu communes. La mortalité y est de $1: 11273$ à $1: 100000$ et a pour principales causes l'infarctus du myocarde, l'embolie pulmonaire, l'insuffisance respiratoire et l'accident vasculaire cérébral. Selon une perspective épidémiologique, la rareté des complications sévères rend les études statistiquement significatives difficiles.

La faible morbidité est devenue, par conséquent, une mesure de substitution lors de l'évaluation des suites de la chirurgie ambulatoire. Les méthodes utilisées pour évaluer la morbidité mineure comprennent les symptômes postopératoires qui précèdent et suivent le départ du patient. Les symptômes les plus fréquents dans les 24 premières heures suivant le départ sont les maux de 
gorge ( $28 \%$ ), les douleurs $(24 \%)$, les céphalées ( $12 \%$, la somnolence (11\%), les nausées et les vomissements (7\%) et la fièvre $(5 \%) .{ }^{5}$ Ces incidences n'ont pas changé depuis 20 ans malgré les modifications de la pratique de la chirurgie et de l'anesthésie! ${ }^{6}$ Les incidences précises de symptômes mineurs vont varier selon l'ensemble des divers cas pris en charge à chaque institution. Certaines opérations, comme la laparoscopie gynécologique et l'amygdalectomie, sont associées à plus de douleur et de vomissements. Des incidences élevées de vomissements, de somnolence et d'étourdissement sont reliées à l'administration d'opiö̈des post opératoires. Le traitement insuffisant de la douleur et des nausées est toujours actuel dans de nombreux hôpitaux même si une grande proportion des patients en souffrent. ${ }^{7}$

Le taux des admissions imprévues à l'hôpital est une autre mesure de ce qui se passe après la chirurgie ambulatoire. Il contribue à régler le contrôle de la qualité et à fournir des données essentielles permettant de déterminer l'association appropriée des opérations et des types de patients. Les séries publiées signalent des taux de $0,1 \%$ à $9,5 \%$ et les unités de chirurgie ambulatoire en milieu hospitalier tendent à présenter des taux d'admission plus élevés. Les raisons habituelles d'admissions imprévues sont les douleurs, les saignements, les vomissements, les évanouissements, les raisons d'ordre social et les complications chirurgicales. Toutefois, il n'y a pas eu d'etude prospective à grande échelle essayant d'identifier les facteurs prédictifs possibles des admissions non prévues. Dans le présent numéro du Journal, Fortier et Chung ${ }^{8}$ décrivent leur étude prospective de 15179 patients de l'hôpital de Toronto. Le taux total d'admission non prévue était de $1,42 \%$. Les chirurgies oto-rhino-laryngologique ( $18 \%)$ et urologique $(4,8 \%)$ présentent les taux les plus élevés. Les interventions longues $(>1 \mathrm{~h})$ et celles qui se terminent après $15 \mathrm{~h}$ étaient plus susceptibles de donner lieu à une admission non planifiée. Les facteurs post opératoires prédictifs d'admission non planifiée ont été les saignements, les douleurs, la somnolence et les nausées et les vomissements. L'étude présente n'a pas conclu que l'anesthésie générale était un important facteur de risque. Gold et coll. ${ }^{9}$ ont considéré l'anesthésie générale comme un facteur de risque selon un coefficient de 5,18. C'est peutêtre un changement dans la pratique de l'anesthésie avec l'emploi d'agents d'action brève, comme le propofol, qui explique cette différence. Des liens inattendus sont apparus comme le sexe mâle et l'indice de la masse corporelle. Intuitivement, cela n'a pas de sens et nécessite un examen ultérieur. Cependant, il est clair que beaucoup de causes de réadmission sont d'ordre social et médical. Ces deux causes soulignent le besoin d'une meilleure éducation du patient et du chirurgien autant que de l'optimalisation des ressources médicales et du dépistage d'un meilleur support social à domicile.

En conclusion, il est troublant que tant de patients externes se plaignent de symptômes mineurs comme les maux de gorge, les céphalées, la somnolence, les douleurs et les maux de dos et expriment un taux élevé d'insatisfaction (65\%). ${ }^{10}$ Un nombre croissant de symptômes apparaissant $24 \mathrm{~h}$ après l'opération est un facteur prédictif d'insatisfaction par rapport à l'anesthésie et d'insatisfaction globale face à la chirurgie ambulatoire. ${ }^{11}$ L'échec à traiter les symptômes habituels comme la douleur peut mener, chez les enfants, à des changements sévères de comportement qui peuvent durer jusqu'à quatre semaines après la chirurgie d'un jour. ${ }^{12}$ Des rapports plus récents de hauts taux d'infection dans le traitement des patients externes sont tout aussi inquiétants et on doit en tenir compte. ${ }^{13}$ Une étude encore plus serrée des séquelles postopératoires chez des patients ambulatoires est tout à fait justifiée et doit ajouter des indicateurs comme le retour aux activités et les complications tardives postchirurgie ambulatoire tant dans les unités affiliées aux l'hôpitaux que dans les établissements autonomes. Au Canada, aucun organisme n'a été mandaté pour la mise en application d'une solution dynamique globale assurant la qualité de traitement du patient externe. Il existe un potentiel d'expansion croissante des établissements autonomes, offrant des services assurés, et d'expansion compétitive de services non assurés. Les autorités provinciales doivent développer une structure globale qui s'occupe de la qualité des soins dans tous les services pour patients externes.

\section{References}

1 Nicoll JH. The surgery of infancy. Br J Med 1909; 2: 753-6.

2 Evans $R G$, Robinson GC. Surgical day care: measurements of the economic payoff. Can Med Assoc J 1980; 123: 873-80.

3 Mitchell $A$. Cutbacks not the only cause of vanishing hospital beds. The Globe and Mail. 1997; April 22: A8.

4 Harrison $M L$, Graff $L A$, Roos NP, et al. Discharging patients earlier from Winnipeg hospitals: does it adversely affect quality of care? Can Med Assoc J 1995; 153: 745-51.

5 Chung F, Un V, Su J. Postoperative symptoms 24 hours after ambulatory anaesthesia. Can J Anaesth 1996; 43: 1121-7.

$6 O_{g g} T W$. An assessment of postoperative outpatient cases. BMJ 1972; 4: 573-5. 
7 Kotiniemi LH, Rybänen PT, Valanne J, Jokela $R$, Mustonen A, Poukkula E. Postoperative symptoms at home following day-case surgery in children: a multicentre survey of 551 children. Anaesthesia 1997; 52: 963-9.

8 Fortier J, Chung F. Unanticipated admission after ambulatory surgery - a prospective study. Can J Anaesth 1998; 45: 612-19.

9 Gold BS, Kitz DS, Lecky JH, et al. Unanticipated admission to the hospital following ambulatory surgery. JAMA $1989 ; 262: 3008-10$.

10 Duncan PG, Cohen MM, Tweed WA, et al. The Canadian four centre study of anaesthetic outcomes: III. Are anaesthetic complications predictable in day surgical practice? Can J Anaesth 1992; 39: 440-8.

11 Tong $D$, Chung $F$, Wong $D$. Predictive factors in global and anesthesia satisfaction in ambulatory surgical patients. Anesthesiology 1997; 87: 856-64.

12 Kotiniemi LH, Rybänen PT, Moilanen IK. Behavioral changes in children following day-case surgery: a 4-week follow-up of 551 children. Anaesthesia 1997; 52: 970-6.

13 Herwaldt LA, Smith $S D$, Carter $C D$. Infection control in the outpatient setting. Infect Control Hosp Epidemiol 1998; 19: 41-74.

14 Lavis JH, Lomas J, Anderson GM, et al. Free-standing health facilities: financial arrangements, quality assurance and a pilot study. Can Med Assoc J 1998; 158: 359-63. 